VOL. 67 (2003) [277-284]

\title{
AUTOCONJUGATE FUNCTIONS AND REPRESENTATIONS OF MONOTONE OPERATORS
}

\author{
JeAn-Paul Penot
}

\begin{abstract}
We show the existence of a convex representation of a maximal monotone operator by a convex function which is invariant with respect to the Fenchel conjugacy (up to an interchange of variables). We use the framework of generalised convexity.
\end{abstract}

\section{INTRODUCTION}

The representations of maximal monotone operators on a reflexive Banach space $X$ by closed proper convex functions on the product $X \times X^{*}$, first obtained by Krauss $[7,8,9,10]$ and Fitzpatrick [4], have recently received a renewed interest. In particular, Martínez-Legaz and Théra [13] have characterised the image of the Fitzpatrick representation and described the inverse correspondence; Burachik and Svaiter [2] have given a criterion ensuring that a convex function on $X \times X^{*}$ represents a maximal monotone operator and have introduced a whole class of such functions [3]. Penot [20] has used a special kind of representations to deduce results about operations on maximal monotone operators from classical results of convex analysis. His approach is connected with the following problem: given a monotone operator $M: X \rightrightarrows X^{*}$, it is possible to get a closed convex function $q$ on $X \times X^{*}$ such that $q^{*}\left(x^{*}, x\right)=q\left(x, x^{*}\right)$ for any $\left(x, x^{*}\right) \in X \times X^{*}$ and $f_{M} \leqslant q \leqslant p_{M}$, where $f_{M}$ is the Fitzpatrick representation of $M$ and $p_{M}=f_{M}^{*}$. A positive answer is provided here in the broader framework of generalised convexity and generalised monotonicity (see $[1,14,15,27,29]$ ). For the study of maximal monotone operators and their representations by convex functions (on spaces which are larger than $X \times X^{*}$ ), we refer to the recent monograph by Simons [28].

\section{Dualities}

Definition 1: Given a pair of sets $W, Y$ a duality is a mapping $D: f \mapsto f^{D}$ from $\overline{\mathbb{R}}^{W}$ into $\overline{\mathbb{R}}^{Y}$ (where $\overline{\mathbb{R}}:=\mathbb{R} \cup\{-\infty,+\infty\}$ ), such that, for any family $\left(f_{i}\right)_{i \in I}$ of $\overline{\mathbb{R}}^{W}$,

$$
\left(\inf _{i \in I} f_{i}\right)^{D}=\sup _{i \in I} f_{i}^{D}
$$

Received 10th September, 2002

Copyright Clearance Centre, Inc. Serial-fee code: 0004-9727/03 \$A2.00+0.00. 
The reverse (or dual or reciprocal) duality $D^{\prime}: \overline{\mathbb{R}}^{Y} \rightarrow \overline{\mathbb{R}}^{W}$ is then defined by

$$
g^{D^{\prime}}:=\inf \left\{h \in \overline{\mathbb{R}}^{W}: h^{D} \leqslant g\right\} .
$$

Then the pair $\left(D, D^{\prime}\right)$ is a Galois correspondence between the complete lattices $\overline{\mathbb{R}}^{W}$ and $\overline{\mathbb{R}}^{Y}$ and one can draw from that useful consequences:

$$
\begin{aligned}
& \forall f \in \overline{\mathbb{R}}^{W} \quad f^{D D^{\prime} D}=f^{D}, \quad \forall g \in \overline{\mathbb{R}}^{Y} \quad g^{D^{\prime} D D^{\prime}}=g^{D^{\prime}} \\
& \forall f \in \overline{\mathbb{R}}^{W} \quad \forall g \in \overline{\mathbb{R}}^{Y} \quad\left(f^{D} \leqslant g\right) \Longleftrightarrow\left(g^{D^{\prime}} \leqslant f\right) \\
& \forall f \in \overline{\mathbb{R}}^{W} \quad\left(f^{D D^{\prime}}=f\right) \Leftrightarrow\left(\exists g \in \overline{\mathbb{R}}^{Y}: f=g^{D^{\prime}}\right) .
\end{aligned}
$$

We denote by $\Gamma_{D}(W)$ (respectively, $\Gamma_{D^{\prime}}(Y)$ ) the image of $D$ (respectively, $D^{\prime}$ ).

Among dualities, a familiar class is formed by conjugacies (or conjugations), that is, dualities for which

$$
(f+r)^{D}=f^{D}-r \quad \forall f \in \overline{\mathbb{R}}^{W} \quad \forall r \in \mathbb{R} .
$$

It can be shown ([12]) that $D$ is a conjugacy if and only if there exists a coupling function $c: W \times Y \rightarrow \overline{\mathbb{R}}:=\mathbb{R} \cup\{-\infty,+\infty\}$ such that $f^{D}=f^{c}$, where

$$
f^{c}(y):=-\inf \{f(w)-c(w, y): w \in W\}
$$

with the convention $(+\infty)+(-\infty)=+\infty$. In such a case, $\Gamma_{D}(W)$ is the set of functions on $W$ which are suprema of families of functions of the form $w \mapsto c(w, y)-r$ for $(y, r)$ in some subset of $Y \times \mathbb{R}$; a similar assertion holds for $\Gamma_{D^{\prime}}(Y)$. Conjugacies have been introduced by Moreau [14] and have been studied by a number of authors (see [1, 6, $11,16,19,18,15,22,23,26]$ and the references therein). It has also be shown by Martínez-Legaz and Singer ([12]) that dualities from $\overline{\mathbb{R}}^{W}$ into $\overline{\mathbb{R}}^{Y}$ are characterised as the mappings $D: \overline{\mathbb{R}}^{W} \rightarrow \overline{\mathbb{R}}^{Y}$ for which there exists a function $G: W \times Y \times \overline{\mathbb{R}} \rightarrow \overline{\mathbb{R}}$ (called the generating function of $D)$ such that for any $(w, y) \in W \times Y$ the function $G(w, y, \cdot)$ is nonincreasing, lower semicontinuous and such that

$$
f^{D}(y)=\sup _{w \in W} G(w, y, f(w)) \quad \forall f \in \overline{\mathbb{R}}^{W} \forall y \in Y .
$$

The generating function $G$ is given by

$$
G(w, y, r):=\left(\iota_{\{w\}}+r\right)^{D}(y)
$$

where $\iota_{S}$ is the indicator function of a subset $S$ of $W$ given by $\iota_{S}(w)=0$ if $w \in S,+\infty$ else. When $D$ is a conjugacy with coupling function $c$, formula (3) is reduced to

$$
G(w, y, r)=-(r-c(w, y))
$$


With any duality one can associate a notion of subdifferential. However, for simplicity, we only consider the case $D$ is a conjugacy arising from a coupling function $c$; then the $c$-subdifferential of a function $f \in \overline{\mathbb{R}}^{W}$ at some point $w \in W$ where $f(w)$ is finite is the set

$$
\partial^{c} f(w):=\{y \in Y: f(\cdot)-c(\cdot, y) \geqslant f(w)-c(w, y)\} .
$$

As in [15, Section 2.4], which deals with the case $c$ is an evaluation function, we observe that the multimapping $M: w \rightrightarrows \partial^{c} f(w)$ is c-monotone in the following sense:

$$
c(w, y)+c\left(w^{\prime}, y^{\prime}\right) \geqslant c\left(w^{\prime}, y\right)+c\left(w, y^{\prime}\right) \quad \forall w, w^{\prime} \in W, y \in M(w), y^{\prime} \in M\left(w^{\prime}\right) .
$$

When $X$ is a Banach space, $W=X, Y:=X^{*}$ and $c: X \times X^{*} \rightarrow \mathbb{R}$ is the natural pairing $c(\cdot, \cdot):=\langle\cdot, \cdot\rangle$, this definition coincides with the usual one. We say that a multimapping $M: W \rightrightarrows Y$ is maximal c-monotone if it is $c$-monotone and if its graph is not strictly contained in the graph of a $c$-monotone operator.

\section{REPRESENTATIONS OF MONOTONE OPERATORS.}

In the sequel, $W$ and $Y$ are sets, $c: W \times Y \rightarrow \overline{\mathbb{R}}$ is a coupling function and we focus our attention on $c$-monotone operators $M: W \rightrightarrows Y$. We set $Z:=W \times Y$ and we consider the duality $D: \overline{\mathbb{R}}^{Z} \rightarrow \overline{\mathbb{R}}^{Z}$, given by $D(f)=f^{D}$, with

$$
f^{D}\left(w^{\prime}, y^{\prime}\right):=-\inf \left\{f\left(w^{\prime}, y^{\prime}\right)-\left(c\left(w, y^{\prime}\right)+c\left(w^{\prime}, y\right)\right):(w, y) \in Z\right\} \quad\left(w^{\prime}, y^{\prime}\right) \in Z .
$$

This duality is the conjugacy associated with the coupling function $c_{D}: Z \times Z \rightarrow \overline{\mathbb{R}}$ given by

$$
c_{D}\left(z, z^{\prime}\right):=c\left(w, y^{\prime}\right)+c\left(w^{\prime}, y\right) \quad \text { for } z:=(w, y), z^{\prime}:=\left(w^{\prime}, y^{\prime}\right) \in Z .
$$

When $W$ is a reflexive Banach space $X, Y=X^{*}$ and $c$ is the evaluation $\left(x, x^{*}\right)$ $\mapsto x^{*}(x)$, this duality is close to the classical Fenchel conjugacy since it is composed of this conjugacy with the interchange of variables $\left(x^{*}, x\right) \mapsto\left(x, x^{*}\right)$. As in such a case, one disposes of the representations

$$
f_{M}:=\left(c_{M}\right)^{D}, \quad p_{M}:=\left(c_{M}\right)^{D D}
$$

of $[4,3,20]$ respectively, where $c: W \times Y \rightarrow \mathbb{R}$ is the given pairing, $c_{M}:=c+\iota_{M}$ and where $M$ is identified with its graph. Part of the interest of these representations is expounded in the following statement.

LEMMA 2. Let $M: W \rightrightarrows Y$ be a $c$-monotone operator. Then

(a) the functions $f_{M}$ and $p_{M}$ belong to $\Gamma_{D}(Z)$ and one has $f_{M} \leqslant p_{M} \leqslant c_{M}$;

(b) if (the graph of) $M$ is contained in the domain of cthen one has $c=f_{M}$ $=p_{M}$ on $M$; 
(c) if $M$ is maximal c-monotone, then one has $c \leqslant f_{M} \leqslant p_{M}$ and $\{z \in Z$ : $\left.f_{M}(z)=c(z)\right\} \subset M$

(d) if $M$ is maximal $c$-monotone and if its graph is contained in the domain of $c$ then one has $c \leqslant f_{M} \leqslant p_{M}$ and $M=\left\{z \in Z: f_{M}(z)=c(z)\right\}=\{z \in Z:$ $\left.p_{M}(z)=c(z)\right\}$.

Proof: (a) The inclusions $f_{M}, p_{M} \in \Gamma_{D}(Z)$ are obvious. Using [14, Proposition 3.c], we deduce from the $c$-monotonicity of $M$ that for any $\left(w^{\prime}, y^{\prime}\right) \in W \times Y,(w, y)$ $\in W \times Y$

$$
c\left(w^{\prime}, y^{\prime}\right)+\iota_{M}\left(w^{\prime}, y^{\prime}\right) \geqslant-\left[c(w, y)+\iota_{M}(w, y)-\left(c\left(w^{\prime}, y\right)+c\left(w, y^{\prime}\right)\right)\right] .
$$

Setting $c_{M}:=c+\iota_{M}$ and taking the supremum over $(w, y) \in W \times Y$ we get

$$
c_{M} \geqslant f_{M}
$$

Taking the biconjugates, we obtain $p_{M} \geqslant f_{M}$.

(b) When $M$ is contained in the domain of $c$, for $\left(w^{\prime}, y^{\prime}\right) \in M$ one can take $(w, y)=\left(w^{\prime}, y^{\prime}\right)$ in the supremum giving $f_{M}$, one can simplify by $c\left(w^{\prime}, y^{\prime}\right)$ and get $f_{M}\left(w^{\prime}, y^{\prime}\right) \geqslant-\left[-c\left(w^{\prime}, y^{\prime}\right)+\iota_{M}\left(w^{\prime}, y^{\prime}\right)\right]=c\left(w^{\prime}, y^{\prime}\right)$. Since $c_{M} \geqslant p_{M} \geqslant f_{M}$, one gets $c\left(w^{\prime}, y^{\prime}\right)=c_{M}\left(w^{\prime}, y^{\prime}\right)=p_{M}\left(w^{\prime}, y^{\prime}\right)=f_{M}\left(w^{\prime}, y^{\prime}\right)$.

(c) If $M$ is maximal $c$-monotone, for any $\left(w^{\prime}, y^{\prime}\right) \in(W \times Y) \backslash M$ one can find some $(w, y) \in M$ such that

$$
c\left(w^{\prime}, y^{\prime}\right)+c(w, y)<c\left(w^{\prime}, y\right)+c\left(w, y^{\prime}\right) .
$$

Then one has $c\left(w^{\prime}, y^{\prime}\right)<+\infty, c_{M}(w, y)=c(w, y)<+\infty$, and

$$
\begin{aligned}
c\left(w^{\prime}, y^{\prime}\right) & <c\left(w^{\prime}, y\right)+c\left(w, y^{\prime}\right)-c(w, y)=-\left[c_{M}(w, y)-\left(c\left(w^{\prime}, y\right)+c\left(w, y^{\prime}\right)\right)\right] \\
& \leqslant \sup \left\{-\left[c_{M}\left(w^{\prime \prime}, y^{\prime \prime}\right)-\left(c\left(w^{\prime}, y^{\prime \prime}\right)+c\left(w^{\prime \prime}, y^{\prime}\right)\right)\right]:\left(w^{\prime \prime}, y^{\prime \prime}\right) \in W \times Y\right\} \\
& \leqslant f_{M}\left(w^{\prime}, y^{\prime}\right) .
\end{aligned}
$$

(d) is a consequence of (b) and (c).

A partial converse of assertion (d) of the preceding lemma can be given. When $W$ is a reflexive Banach space, $Y=W^{*}$ and $c$ is the evaluation map given by $c(w, y)=y(w)$, a full converse is displayed in [2].

LEMmA 3. Suppose $W$ and $Y$ are convex subsets of some vector spaces and $Z$ $:=W \times Y$. If $g: Z \rightarrow \overline{\mathbb{R}}$ is a convex function such that $g \geqslant c$, and if the coupling function $c$ takes finite values and is concave in both variables, then $M:=\{z: g(z)=c(z)\}$ is $c$-monotone. 
Proof: Let $z:=(w, y) \in M, z^{\prime}:=\left(w^{\prime}, y^{\prime}\right) \in M$. Then

$$
\begin{aligned}
\frac{1}{2} c(w, y)+\frac{1}{2} c\left(w^{\prime}, y^{\prime}\right) & =\frac{1}{2} g(w, y)+\frac{1}{2} g\left(w^{\prime}, y^{\prime}\right) \\
& \geqslant g\left(\frac{1}{2}(w, y)+\frac{1}{2}\left(w^{\prime}, y^{\prime}\right)\right) \geqslant c\left(\frac{1}{2}\left(w+w^{\prime}\right), \frac{1}{2}\left(y+y^{\prime}\right)\right) \\
& \geqslant \frac{1}{4} c(w, y)+\frac{1}{4} c\left(w, y^{\prime}\right)+\frac{1}{4} c\left(w^{\prime}, y\right)+\frac{1}{4} c\left(w^{\prime}, y^{\prime}\right),
\end{aligned}
$$

so that $c(w, y)+c\left(w^{\prime}, y^{\prime}\right) \geqslant c\left(w^{\prime}, y\right)+c\left(w, y^{\prime}\right): M$ is $c$-monotone.

The preceding proof is similar to an argument due to Martínez-Legaz and Svaiter; see also [20, Proposition 3] in which its origins are described. The assumptions on $c$ are satisfied in each of the following examples.

EXAMPLE 1. $W$ is a normed vector space, $Y=W^{*}$ and the coupling function $c$ is given by $c(w, y):=\langle y, w\rangle-k(w)$, where $k: W \rightarrow \mathbb{R}$ is convex. The case $k(\cdot)=(1 / 2) r\|\cdot\|^{2}$ corresponds to the classical theory of augmented Lagrangians $([\mathbf{6}, 25])$; some extensions to more general situations are given in $[\mathbf{1}, \mathbf{1 8}, \mathbf{2 3}, \mathbf{2 6}]$.

EXAMPLE 2. $W$ is a normed vector space, $Y=W^{*}$ and the coupling function $c$ is given by $c(w, y):=\min (\langle y, w\rangle, 0)$, an important case for quasiconvex programming $([11,17$, 22, 24]).

EXAMPLE 3. $W$ is a normed vector space, $Y=W^{*} \times \mathbb{R}$ and the coupling function $c$ is given by $c\left(w,\left(w^{*}, r\right)\right):=\min \left(\left\langle w^{*}, w\right\rangle, r\right)$, which is also important for quasiconvex duality $([11,16,21])$.

EXAMPLE $4 . W$ is a normed vector space, $Y=W^{*} \times L_{+}\left(W, W^{*}\right)$ where $L_{+}\left(W, W^{*}\right)$ is the cone of positive semidefinite symmetric operators from $W$ into $W^{*}$ and $c\left(w,\left(w^{*}, A\right)\right)$ $=\left\langle w^{*}, w\right\rangle-1 / 2\langle A w, w\rangle([\mathbf{5}])$.

The representation we have in view is given in the following statement.

THEOREM 4. For any $c$-monotone operator $M: W \rightrightarrows Y$ there exists $q \in \Gamma_{D}(W$ $\times Y$ ) such that $q^{D}=q$ and $f_{M} \leqslant q \leqslant p_{M}$. If moreover $M$ is maximal $c$-monotone and contained in the domain of $c$, then $M=\{(w, y): q(w, y)=c(w, y)\}$.

COROLlary 5. ([20]) For any reflexive Banach space $X$ and any maximal monotone operator $M: X \rightrightarrows X^{*}$ there exists a closed convex function $q: X \times X^{*} \rightarrow \overline{\mathbb{R}}$ such that $q^{*}\left(x^{*}, x\right)=q\left(x, x^{*}\right)$ for any $\left(x, x^{*}\right) \in X \times X^{*}$ and $f_{M} \leqslant q \leqslant p_{M}$. If moreover $M$ is maximal monotone then $M=\left\{\left(x, x^{*}\right): q\left(x, x^{*}\right)=\left\langle x^{*}, x\right\rangle\right\}$.

The theorem is a consequence of Lemma 2 and of the following proposition inspired by [9] Theorem 4 and [20] Proposition 10. Let us note that here too uniqueness of $q$ is not ensured. However, as claimed by the second assertion, the coincidence set of $q$ and $c$ is independent of the choice of $q$ when $M$ is maximal monotone; this assertion stems from the fact that this set is also the coincidence set of $p_{M}$ with $c$ and of $p_{M}^{D}$ with $c$. 
In the following proposition which casts the preceding statement in a more general framework, $Z$ is any set and $D: \overline{\mathbb{R}}^{Z} \rightarrow \overline{\mathbb{R}}^{Z}$ is a duality satisfying the conditions:

(A) $\left((1 / 2) q+(1 / 2) q^{D}\right)^{D} \leqslant(1 / 2) q+(1 / 2) q^{D}$ for any function $q \in \overline{\mathbb{R}}^{Z}$ such that $q^{D} \leqslant q$;

(B) for any $z \in Z$ one has $\limsup _{r \rightarrow \infty}\left(r-\left(\iota_{\{z\}}+r\right)^{D}(z)\right)>0$,

Condition (B) can be rephrased in terms of the generating function $G$ of $D$ as:

$\left(\mathrm{B}^{\prime}\right)$ for any $z \in Z$ one has $\limsup _{r \rightarrow \infty}(r-G(z, z, r))>0$.

It is satisfied when $D$ is the conjugacy induced by a coupling $c$ such that $c(z, z)$ is finite for each $z \in Z$, since then $\iota_{z}^{D}(z)=c(z, z)$ and $(f+r)^{D}=f^{D}-r$ for any $f \in \overline{\mathbb{R}}^{Z}$ and any $r \in \mathbb{R}$ (or since then $r-G(z, z, r)=2 r-c(z, z)$ ). Since $G(z, z, \cdot)$ is nonincreasing, it is also satisfied when the following condition is fulfilled:

$\left(\mathrm{B}^{\prime \prime}\right) \quad$ for any $z \in Z$ there exists some $r \in \mathbb{R}$ such that $G(z, z, r)<+\infty$.

Condition (A) is also satisfied whenever $D$ is the conjugacy induced by a coupling $c$. More generally, condition (A) is satisfied when the generating function $G$ associated with the duality $D$ is convex in its third variable. Then $D$ satisfies the following convexity property:

$\left(\mathrm{A}^{\prime}\right)$ for any $f, g \in \overline{\mathbb{R}}^{Z}, s, t \in \mathbb{R}_{+}$with $s+t=1$ one has $(s f+t g)^{D} \leqslant s f^{D}+t g^{D}$. In fact, for any $f, g \in \overline{\mathbb{R}}^{Z}, s, t \in \mathbb{R}_{+}$with $s+t=1$ and for any $z \in Z$ one has

$$
\begin{aligned}
(s f+t g)^{D}(z) & =\sup _{w \in Z} G(w, z, s f(w)+t g(w)) \\
& \leqslant s \sup _{w \in Z} G(w, z, f(w))+t \sup _{w^{\prime} \in Z} G\left(w^{\prime}, z, g\left(w^{\prime}\right)\right) \\
& \leqslant s f^{D}(z)+t^{D}(z) .
\end{aligned}
$$

Proposition 6. Let $Z$ be an arbitrary set and let $D: f \mapsto f^{D}, D: \overline{\mathbb{R}}^{Z} \rightarrow \overline{\mathbb{R}}^{Z}$ be a duality satisfying conditions $(A)$ and (B). Let $p: Z \rightarrow \mathbb{R} \cup\{+\infty\}$ be such that $p \geqslant p^{D}$. Then there exists a function $q: Z \rightarrow \mathbb{R} \cup\{+\infty\}$ such that $q^{D}=q$ and $p \geqslant q \geqslant p^{D}$.

The first part of the following proof is a simplified form due to C. Zalinescu of a proof given in a preliminary version of the paper [20]. The second part fills a gap disclosed by B.F. Svaiter while reading a draft of [20].

PROOF: Let $R$ be the set of functions $r: Z \rightarrow \mathbb{R} \cup\{+\infty\}$ such that $r^{D D}=r, p \geqslant r$ $\geqslant r^{D} \geqslant p^{D}$. This set is nonempty as $p^{D D} \in R$. Let us show that $R$ is (downward) inductive for the pointwise order. Given a totally ordered family $\left(r_{i}\right)_{i \in I}$, let $r:=\left(\inf _{i \in I} r_{i}\right)^{D D}$. Then $r$ is such that $p \geqslant r \geqslant p^{D}$ and $r^{D}=\left(\inf _{i \in I} r_{i}\right)^{D}=\sup _{i \in I} r_{i}^{D}=\lim _{i \in I} r_{i}^{D}$, so that $r^{D} \leqslant \inf _{i \in I} r_{i}$. Taking biconjugates, we get $r^{D} \leqslant r$ and $r \in R$ is a minorant of $\left(r_{i}\right)_{i \in I}$. By Zorn lemma, $R$ has a minimal element $q$. Let us show that $q^{D}=q$. Let $r=(1 / 2) q+(1 / 2) q^{D} \leqslant q$. 
Then $r^{D} \geqslant q^{D} \geqslant p^{D}$. On the other hand, by assumption (A),

$$
r^{D}(z) \leqslant \frac{1}{2} q^{D}(z)+\frac{1}{2} q(z)=r(z) .
$$

Thus $r \in R$. Since $r \leqslant q$, we get $r=q$, hence $q=q^{D}$ on dom $q$.

Suppose now that there exists some $a \in Z \backslash \operatorname{dom} q$ such that $q^{D}(a)<+\infty$. Let $\alpha \geqslant q^{D}(a)$ and let $s: Z \rightarrow \mathbb{R} \cup\{+\infty\}$ be defined by $s(a):=\alpha, s(z):=+\infty$ for $z \neq a$. Taking $\alpha$ large enough, by assumption (B), we may suppose that $s^{D}(a) \leqslant \alpha$. Thus $s^{D} \leqslant s$ and since $s \geqslant q^{D}$ we also have $s^{D} \leqslant q$. Let $t:=(\min (q, s))^{D D}$. Then $t \leqslant q \leqslant p$ and $t^{D}=(\min (q, s))^{D}=\max \left(q^{D}, s^{D}\right) \leqslant q$; we also have $\max \left(q^{D}, s^{D}\right) \leqslant s$, so that $t^{D} \leqslant \min (q, s)$. Taking biconjugates, we get $t^{D} \leqslant t: t \in R$. Since $t \leqslant q$, the minimality of $q$ implies that $t=q$. Since $t(a) \leqslant \alpha<+\infty=q(a)$, we get a contradiction. Thus $q^{D}=q$ everywhere.

\section{REFERENCES}

[1] E.J. Balder, 'An extension of duality-stability relations to nonconvex problems', SIAM J. Control Optim. 15 (1977), 329-343.

[2] R.S. Burachik and B.F. Svaiter, 'Maximal monotonicity, conjugation and the duality product', (preprint, IMPA Rio, October 2001).

[3] R.S. Burachik and B.F. Svaiter, 'Maximal monotone operators, convex functions and a special family of enlargements', Set-Valued Anal. (2002) (to appear).

[4] S. Fitzpatrick, 'Representing monotone operators by convex functions', in Functional Analysis and Optimization, (workshop and miniconference, Canberra, Australia 1988), Proc. Center Math. Anal. Austral. Nat. Univ. 20 (Australian National University, Canberra ACT, 1988), pp. 59-65.

[5] A.D. Ioffe, 'Abstract convexity and non-smooth analysis', in Advances in Mathematical Economics 3 (Springer-Verlag, Tokyo, 2001), pp. 45-61.

[6] R. Janin, 'Sur la dualité en programmation dynamique', C.R. Acad. Sci. Paris A 277 (1973), 1195-1197.

[7] E. Krauss, 'On the maximality of the sum of monotone operators', Math. Nachr. 101 (1981), 199-206.

[8] E. Krauss, 'A representation of maximal monotone operators by saddle functions', Rev. Roumaine Math. Pures Appl. 30 (1985), 823-836.

[9] E. Krauss, 'A representation of arbitrary maximal monotone operators via subgradients of skew-symmetric saddle functions', Nonlinear Anal. 9 (1985), 1381-1399.

[10] E. Krauss, 'Maximal monotone operators and saddle functions. I', Z. Anal. Anwendungen 5 (1986), 333-346.

[11] J.-E. Martínez-Legaz, 'On lower subdifferentiable functions', in Trends in Mathematical Optimization, Internat. Series Numer. Math. 84 (Birkhauser, Basel, 1988), pp. 197-232.

[12] J.-E. Martínez-Legaz and I. Singer, 'Subdifferentials with respect to dualities', ZOR-Math. Methods Oper. Res. 42 (1995), 109-125.

[13] J.E. Martínez-Legaz and M. Théra, 'A convex representation of maximal monotone operators', J. Nonlinear Convex Anal. 2 (2001), 243-247. 
[14] J.-J. Moreau, 'Inf-convolution, sous-additivité, convexité des fonctions numériques', $J$. Math. Pures Appl. 49 (1970), 109-154.

[15] D. Pallaschke and S. Rolewicz, Foundations of mathematical optimization, Mathematics and its Applications 38 (Kluwer, Dordrecht, 1998).

[16] J.-P. Penot, 'What is quasiconvex analysis?', Optimization 47 (2000), 35-110.

[17] J.-P. Penot, 'A Lagrangian approach to quasiconvex analysis', (preprint, Univ. of Pau 1998), J. Optim. Theory Appl. 117 (2003) (to appear).

[18] J.-P. Penot, 'A ugmented Lagrangians, duality and growth conditions', J. Nonlinear Convex Anal. (to appear).

[19] J.-P. Penot, 'Rotundity, smoothness and duality', (preprint, Univ. of Pau, October 2001).

[20] J.-P. Penot, 'The relevance of convex analysis for the study of monotonicity', (preprint, Univ. of Pau, April 2002).

[21] J.-P. Penot and M. Volle, 'Another duality scheme for quasiconvex problems', in Trends in Mathematical Optimization, Internat. Series Numer. Math. 84 (Birkhauser, Basel, 1988), pp. 259-275.

[22] J.-P. Penot and M. Volle, 'On quasi-convex duality', Math. Oper. Res. 15 (1990), 597-625.

[23] J.-P. Penot and M. Volle, On strongly convex and paraconvex dualities, in Generalized convexity and fractional programming with economic applications, (A. Cambini, E. Castagnoli, L. Martein, P. Mazzoleni, S. Schaible, Editors), Lecture notes in Econom. and Math. Systems 345, (Proceedings, Pisa, Italy 1988) (Springer-Verlag, Berlin, 198-218).

[24] J.-P. Penot and M. Volle, 'Surrogate programming and multipliers in quasiconvex programming', (preprint, Univ. of Avignon, 1997).

[25] R.T. Rockafellar, 'Augmented Lagrange multiplier functions and duality in nonconvex programming', SIAM J. Control Optim. 12 (1974), 268-285.

[26] R.T. Rockafellar and R.J.-B. Wets, Variational analysis (Springer-Verlag, Berlin, 1998).

[27] A.M. Rubinov, Abstract convexity and global optimization (Kluwer, Dordrecht, 2000).

[28] S. Simons, Minimax and monotonicity, Lecture Notes in Mathematics 693 (Springer-Verlag, Berlin, 1998).

[29] I. Singer, Abstract convex analysis (J. Wiley and Sons, New York, 1997).

Laboratoire de Mathématiques Appliquées

CNRS ERS 2055

Faculté des Sciences

Université de Pau

BP 1155, 64013 PAU Cedex

France 